\title{
AKKUS ERSTER WAHL
}

\section{Liebe Leserin, lieber Leser,}

bei der Auswahl von Traktionsbatterien, genauer deren Zellen, sind die Würfel gefallen. Denn die Autohersteller haben sich entschieden, welche Zellbauformen und chemischen Rezepturen in der ersten Generation ihrer elektrifizierten Antriebe zum Einsatz kommen. Dafür müssen nun die Zellfabriken zuverlässig und in größeren Mengen liefern. Das gelingt aber nicht immer, denn die Fertigungstechniken bestimmen die Spielregeln, und die durchschauen nur wenige.

Gute Karten haben Volkswagen, Ford und Toyota. Sie haben sich mit Zellen von Panasonic für das stabile prismatische Zelldesign entschieden. Diese Bauform ist zwar schwerer und größer als das konkurrierende Pouchzellendesign. Doch viele Experten sind trotz des besseren Package der Pouchzellen skeptisch - oft wird diskutiert, ob die Bauform Schuld daran ist, dass dieser Zelltyp in puncto Dauerhaltbarkeit wie bei Nissan versagt hat. In den Batterien des OEM sind bereits nach zwei anstatt der geforderten zehn Jahre nur noch 80 \% Maximalkapazität nutzbar.

Kein schlechtes Blatt haben die genannten OEM und auch andere Hersteller, die auf die Elektrodenmaterialien NickelMangan-Cobalt setzen, und das, obwohl diese Zellen im Vergleich zu LithiumEisenphosphat-Zellen weniger Leistung abgeben können. Kauft VW damit Zellen zweiter Wahl? Nein.

Die Wolfsburger gehen „auf Nummer sicher". Denn es gibt Probleme bei der zwar intrinsisch sicheren aber fertigungstechnisch kritischen LithiumEisenphosphat-Zelle. Hier erzeugen kleinste Metallpartikel in den Folien immer wieder Kurzschlüsse. Das musste der US-amerikanische Pionier A123 ebenso leidvoll erfahren wie chinesische Batteriehersteller.
Schwarzmalerei ist dennoch nicht angebracht. Es handelt sich hier schließlich um eine Momentaufnahme - Forschung sowie Entwicklung stehen gemessen an der jungen E-Auto-Historie ja noch am Anfang. Zu den Pionieren zählen unter anderem die Materialwissenschaftler und Produktionstechniker, die Ihnen in dieser Ausgabe der ATZelektronik vorausschauende Ansätze vorstellen. Aus diesen lässt sich die Reife der deutschen Aktivitäten ein Stück weit herauslesen.

Dabei bleiben durchaus Fragen offen. Beispielsweise die nach der Reife des proklamierten Zellfertigungsstandorts Deutschland. Einige Insider sagen, der Aufbau des notwendigen Know-hows dauert noch mehr als zehn Jahre. Man sei Panasonic dicht auf den Fersen, kontern manche. Wie groß ist der Vorsprung der Japaner wirklich? Einige unter ihnen melden ihre Rezepturen erst gar nicht zum Patent an, damit ihnen keiner auf die Schliche kommt. OEM-Kunden kaufen damit eine Blackbox - und fahren gut damit.

Herzliche Grüße

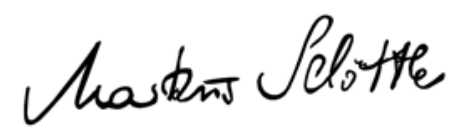

\section{MARKUS SCHÖTTLE,}

Stellvertretender Chefredakteur,

Wiesbaden, 24. April 2013

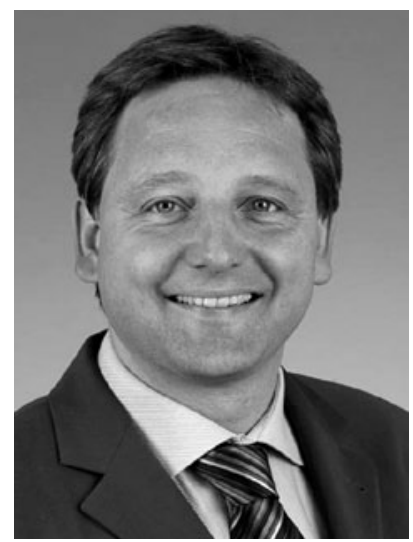

DYNA4 Advanced Powertrain

\section{Energiemanagement im Fahrzeug}

- Simulation von Einzelfahrzeugen bis hin zur Fahrzeugflotte

- Fahrleistungs- und Verbrauchsberechnungen

- Konventionelle Antriebe, Hybridund Elektrofahrzeuge

- Variantenhandling, Automation und Reporting

Mehr erfahren unter www.tesis-dynaware.com/flv 